\title{
Improving Resolution of HAADF STEM Images with Maximum Entropy Method and Deconvolution Processing
}

\author{
Y. Kotaka, ${ }^{*}$ N. Nakanishi, ${ }^{* *}$ T. Yamazaki, ${ }^{* *}$ K. Watanabe, ${ }^{* * *}$ and I. Hashimoto**
}

* Fujitsu Laboratories Ltd., 10-1 Morinosato-Wakamiya, Atsugi, Kanagawa 243-0197, Japan

** Department of Physics, Tokyo University of Science, 1-3 Kagurazaka, Shinjuku, Tokyo 162-8601, Japan

*** Tokyo Metropolitan College of Technology, 1-10-40, Higashi-Ohi, Shinagawa, Tokyo, 140-0011, Japan

High-angle annular dark field (HAADF) scanning transmission electron microscopy (STEM) has been an attractive method for an atomic-scale structural and compositional analysis, because the image has been gained at atomic resolution and its contrast depends on atomic number [1]. To a good approximation, the HAADF STEM images are represented by convolution between the intensity of the probe function and the object function which may be regarded as the projecting atomic structure. By using this approximation, deconvolution processing can extract a clear atomic structure on a relatively thin specimen from an obscure experimental image [2].

Noise reduction is, however, indispensable for the precise analysis of the HAADF STEM images, because the experimental images are noisier than conventional high-resolution electron microscopy images. Fourier filtering method, smoothing method and maximum entropy method (MEM) [3] have commonly been used for noise reduction. These methods have some artificial parameters. In the Fourier filtering, it is difficult to optimize parameters, such as mask size, mask shape and threshold value, for the image including defects. Furthermore, artifacts tend to appear due to noise cut. These problems become more severe in the deconvolution processing. The smoothing method, which is based on averaging, depends on mask size, and makes resolution poor due to averaging. The MEM has parameters such as the standard deviation of noise at each pixel and Lagrange multiplier constants. In this paper, in order to eliminate noise without artificial parameters, the MEM has extended to experimental incoherent images. Furthermore, improving resolution of HAADF STEM images is proposed by combining the MEM with deconvolution processing.

As mentioned above, the MEM has artificial parameters such as standard deviation of noise $\sigma$ and Lagrange multiplier constant $\lambda$. In our method, the standard deviation of nose is estimated using information limit for an incoherent STEM image. The information outside this limit is regarded as noise only. Figure 1 shows an experimental HAADF STEM image of $\mathrm{SrTiO}_{3}$ [001] and the estimated noise. Inset displays the diffractogram of Fig. 1(a) and information limit. Thus, the standard deviation of the noise $\sigma$ can be estimated. Since optimal value of Lagrange multiplier constant $\lambda$ depends on material, magnification, signal-to-noise ratio and other variables, this optimal 
value has to be determined trial-and-error. However, a small deviation from the optimal value does not lead to a false image, unlike an incorrect mask size in the Fourier filtering method. Therefore, the optimal choice is the smallest value of $\lambda$ among the values that converge.

Figure 2 (a) shows the MEM processed image of Fig. 1(a) at $\lambda=1.0$. This image becomes clear with reduced noise. Projected model of this image is also shown in Fig. 2(a). The large, middle and small circles mark $\operatorname{Sr}(Z=38)$, Ti $(Z=22)$ and $\mathrm{O}(Z=8)$ columns, respectively. Reduced noise makes each atomic column stand out while $\mathrm{O}$ columns cannot be detected in this image because of oxygen's small atomic number. Figure 2(b) shows the deconvoluted image of Fig. 2(a). It is found that resolution of image is improved by deconvolution processing. In particular, Ti site is resolved clearly. Thus, it is concluded that this processing composed of the MEM and deconvolution makes the experimental HAADF STEM image clear and enables us to analyze the image in detail.

This method can be useful for noise elimination and resolution improvement in high-resolution HAADF STEM images and helps us to analyze them quantitatively.

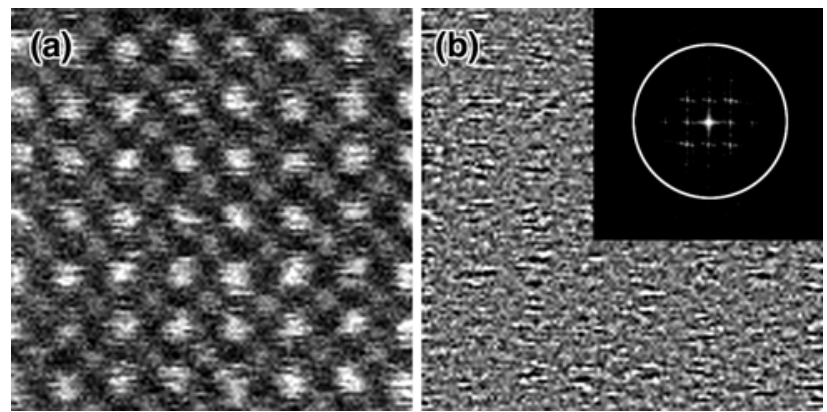

Fig. 1 Experimental HAADF STEM image of $\mathrm{SrTiO}_{3}$. (b) Estimated nose. Inset shows diffractogram of (a) and information limit.
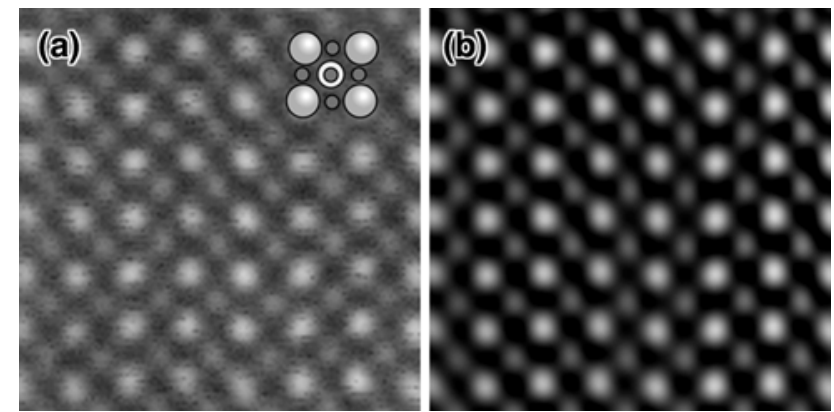

Fig. 2(a) MEM processed image and projected atomic model. (b) Deconvoluted image of (a).

References

[1] S. J. Pennycook and P. D. Nellist, Impact of Electron and Scanning Probe Microscopy on Materials Research, pp. 161-207, Kluwer Academic, Netherlands, 1999.

[2] K. Watanabe et al., Ultramicroscopy, 92 (2002) 191.

[3] S. F. Gull and G. J. Daniel, Nature, 272 (1978) 686.

[4] One of authors (N. N.) is financially supported by the Japan Society for the Promotion of Science (JSPS) as JSPS Research Fellowships for Young Scientists. 